\title{
Motivos para recusa da vacina contra o Papilomavírus Humano entre adolescentes de 11 a 14 anos no município de Maringá-PR
}

Reasons for refusal of human papillomavirus vaccine among adolescent girls between 11 and 14 years of age in the municipality of Maringa-PR

Razones para rechazo de la vacuna contra el virus del papiloma humano en adolescentes entre 11 y 14 años en el municipio de Maringá-PR

Natalie Vieira Zanini ${ }^{a}$, Bianca Stawinski Prado ${ }^{a}$, Rafael de Castro Hendges ${ }^{a}$, Carolina Arnaut dos Santos ${ }^{a}$, Fernanda Vieira Rodovalho Callegarib, Marcelo Picinin Bernucia

\section{Resumo}

Introdução: O Ministério da Saúde incluiu a vacina contra o Papilomavírus Humano (HPV) no calendário básico de imunizações em 2014 com a meta vacinar pelo menos $80 \%$ das adolescentes entre 11 e 13 anos de idade. Objetivos: Frente à baixa adesão à campanha nacional de vacinação contra o HPV no município de Maringá, PR, em 2014, o presente estudo teve como objetivos: 1) identificar o nível de conhecimento das adolescentes acerca do vírus e da vacina; e 2) descrever os motivos pelos quais elas não se vacinaram. Métodos: Trata-se de um estudo observacional transversal, do tipo inquérito domiciliar com delineamento descritivo, conduzido com 58 adolescentes que não tomaram a $1^{\underline{a}}$ e/ou a $2^{\underline{a}}$ dose da vacina dentro do prazo estipulado pela campanha vacinal de 2014 . Para coleta de dados, foi aplicado questionário por meio de entrevistas face a face. Resultados: $86 \%$ das adolescentes já ouviram falar do vírus, $74 \%$ sabe que sua transmissão é sexual, $49 \%$ conhece sua relação com o câncer de colo de útero e $88 \%$ já ouviu falar da vacina. A maior parte das meninas entrevistadas não se vacinou por recusa por parte dos pais. Conclusão: Os resultados do presente estudo sugerem que o desconhecimento sobre a infecção pelo HPV e sobre a campanha de vacinação entre as entrevistadas e seus responsáveis, bem como o medo de efeitos colaterais da vacina, como apontado pela população do estudo, interferiram negativamente na tomada de decisão.
Como citar: Zanini NV, Prado BS, Hendges RC, Santos CA, Rodovalho-Callegari FV, Bernuci MP. Motivos para recusa da vacina contra o Papilomavírus Humano entre adolescentes de 11 a 14 anos no município de Maringá-PR. Rev Bras Med Fam Comunidade. 2017;12(39):1-13. http://dx.doi.org/10.5712/rbmfc12(39)1253
Palavras-chave: Neoplasias do Colo do Útero Doenças Sexualmente Transmitidas Papillomaviridae Sistema Único de Saúde Vacinas contra Papillomavirus
Fonte de financiamento: O estudo foi financiado pelo Conselho Nacional de Desenvolvimento Científico e Tecnológico (CNPq; Projeto Universal No 455495/2014-9). Marcelo Picinin Bernuci recebeu bolsa de auxílio a pesquisa do Instituto Cesumar de Ciência, Tecnologia e Inovação (ICETI). Natalie Vieira Zanini recebeu bolsa de iniciação científica PIBIC-CNPq.

Parecer CEP:

879.619 (UniCesumar), aprovado em 20/11/2014. Conflito de interesses: declaram não haver. Procedência e revisão por pares: revisado por pares. Recebido em: 17/12/2015. Aprovado em: 11/07/2017. 


\begin{abstract}
Introduction: The Ministry of Health included the human papillomavirus (HPV) vaccine in the basic immunization calendar in 2014 with the aim to vaccinate at least $80 \%$ of adolescents aged $11-13$ years old. Objectives: Faced with poor adherence to the national campaign of vaccination against HPV in Maringa, State of Parana, in 2014, this study aimed to: 1) identify the level of knowledge of adolescents about the virus and the vaccine; and 2) describe the reasons why they did not vaccinate. Methods: This is a cross-sectional observational study, a household survey with descriptive design, conducted with 58 adolescents who did not take the first and/or second dose of the vaccine within the period stipulated by the vaccination campaign of 2014. For data collection, a questionnaire was applied through face to face interviews. Results: $86 \%$ of teens have heard of the virus, $74 \%$ know that its transmission is sexual, $49 \%$ know their relationship with cervical cancer and $88 \%$ have heard of the vaccine. Most of the interviewed girls didn't vaccinate due to their parents' refusal Conclusion: The results of this study suggest that lack of knowledge about HPV infection and the vaccination campaign among the respondents and their parents, as well as the fear of vaccine side effects, as pointed out by the study population, interfered negatively in the decision making.
\end{abstract}

\section{Resumen}

Introducción: El Ministerio de Salud incluyó la vacuna contra el papiloma humano (VPH) en el calendario básico de vacunación en 2014 con el objetivo de vacunar al menos el $80 \%$ de los adolescentes de entre 11 y 13 años. Objetivos: Ante la baja adherencia a la campaña nacional de vacunación contra el virus del VPH en Maringá, estado de Paraná, en 2014, este estudio tuvo como objetivo: 1) identificar el nivel de conocimientos de los adolescentes sobre el virus y la vacuna; y 2) describir las razones por las que no están vacunados. Métodos: Se trata de un estudio transversal observacional, una encuesta domiciliaria con un diseño descriptivo, realizado con 58 adolescentes que no tomaron la primera y/o segunda dosis de la vacuna en el plazo estipulado por la campaña de vacunación de 2014. Para la recolección de datos, se aplicó un cuestionario a través de entrevistas cara a cara. Resultados: $86 \%$ de los adolescentes ha oído hablar de los virus, $74 \%$ sabe que su transmisión es sexual, $49 \%$ conoce su relación con el cáncer cervical y $88 \%$ ha oído hablar de la vacuna. La mayoría de las niñas entrevistadas no está vacunada debido al rechazo de los padres. Conclusión: Los resultados de este estudio sugieren que la falta de conocimiento acerca de la infección por VPH y la campaña de vacunación entre los encuestados y sus padres, así como el temor a los efectos secundarios de las vacunas, como se ha señalado por la población de estudio, interfirió negativamente en la toma de decisión.

\section{Keywords:}

Uterine Cervical Neoplasms

Sexually Transmitted

Diseases

Papillomaviridae

Unified Health System

Papillomavirus Vaccines
Palabras clave:

Neoplasias del Cuello Uterino

Enfermedades de

Transmisión Sexual

Papillomaviridae

Sistema Único de Salud

Vacunas contra

Papillomavirus

\section{Introdução}

A infecção pelo Papilomavírus Humano (HPV) é a infecção sexualmente transmissível (IST) mais prevalente no mundo; ${ }^{1}$ acomete a maioria das mulheres sexualmente ativas em algum momento de suas vidas, sendo o risco de contrair a infecção de 50-80\% ao longo da vida. ${ }^{2}$ A relevância da contaminação pelo HPV reside na sua íntima relação com o câncer de colo do útero, ${ }^{2-4}$ terceiro tipo de câncer mais comum entre as mulheres no mundo ${ }^{5}$ e, no Brasil, a terceira causa de morte por câncer na população feminina. ${ }^{6}$ Além do câncer de colo do útero, o HPV tem relação com cânceres da cavidade oral, da orofaringe e anorretal $^{7}$ e com lesões benignas, como as verrugas genitais. ${ }^{8}$

Frente a esse cenário, foi desenvolvida a vacina anti-HPV com intuito profilático, primeiramente a quadrivalente Gardasil, produzida pela Merck e liberada pelo US Food and Drug Administration (FDA) em $2006^{9}$ e, no mesmo ano, pela Agência Nacional de Vigilância Sanitária (ANVISA) no Brasil, para distribuição privada; e posteriormente, a bivalente Cervarix, produzida pela GSK. ${ }^{10}$ Mais recentemente, no final de 2014, foi desenvolvida a vacina nona-valente, ainda não disponível no Brasil. ${ }^{11}$

Apesar de não evitarem totalmente a infecção pelo HPV, uma vez que a imunização não abrange todos os tipos de vírus, mas sim os mais comuns - 16,18,11 e 6 para a Gardasil e 16 e 18 para a Cervarix os resultados têm sido satisfatórios, mostrando que as duas vacinas são seguras, bem toleradas e efetivas na prevenção da infecção pelo HPV e da sua persistência. ${ }^{12}$ Quando provocam efeitos colaterais, eles se resumem a dor e edema na região da aplicação, dor de cabeça, febre e vômito, sendo raros e passageiros. ${ }^{12}$ 
Atualmente, a vacina contra o HPV representa a primeira medida para profilaxia do câncer cervical; no entanto, uma vez que ela não protege contra todos os sorotipos de alto risco oncogênico, para a prevenção deste câncer não basta a vacinação, faz-se necessário o rastreamento pelo exame Papanicolaou (colpocitologia oncótica cervical). ${ }^{13}$

No Brasil, a implementação da vacina anti-HPV pelo Sistema Único de Saúde (SUS) é extremamente recente, colocando inicialmente a vacina do tipo quadrivalente à disposição de meninas entre 11 e 13 anos. ${ }^{14}$ A primeira campanha nacional foi realizada entre 10 de março e 14 de abril de 2014, tendo como meta a vacinação de 4,2 milhões de meninas, número correspondente a $80 \%$ das meninas entre 11 e 13 anos. ${ }^{15}$

No estado do Paraná a meta era vacinar 218.300 meninas, entretanto, até 3 dias do término da campanha, apenas 158 mil (72\%) tinham comparecido a alguma das Unidade Básicas de Saúde (UBS) ou à Secretaria Municipal de Saúde para receber a vacina, ficando o estado abaixo da média nacional $(83 \%) .{ }^{14}$ Dados não oficiais da Secretaria de Saúde de Maringá, PR, divulgados pela mídia ${ }^{16}$ mostraram que no município de Maringá, a uma semana para o fim da campanha, somente 3.947 meninas haviam sido vacinadas, representando $52 \%$ da meta estipulada em 7.532 garotas, o que estava muito aquém do preconizado.

Pesquisas realizadas em diversos países mostraram que fatores de recusa à vacina são muito diversos. ${ }^{17-24}$ Dentre estes, se destacam a necessidade de maiores informações acerca da vacina, medo de possíveis efeitos colaterais, dúvidas quanto a sua eficácia, possível indução de iniciação sexual precoce e, ainda, crença de que o Papanicolaou seja eficiente e suficiente na prevenção do HPV.

Frente à baixa adesão à vacinação contra o HPV no município de Maringá na campanha nacional de 2014, descrevemos no presente estudo os motivos pelos quais as meninas não se vacinaram. Além disso, foi investigado o nível de conhecimento dessa população acerca da relação entre HPV e o câncer de colo do útero e a influência deste conhecimento na tomada de decisão de aderir à campanha de vacinação. Acreditamos que os resultados de nosso estudo possam contribuir para o delineamento de estratégias mais eficazes a serem empregadas na mobilização da população alvo a aderir às campanhas futuras.

\section{Métodos}

Trata-se de um estudo observacional de corte transversal, do tipo inquérito domiciliar com delineamento descritivo, conduzido num grupo de meninas residentes no município de Maringá, PR, entre abril e junho de 2015.

O estudo foi previamente aprovado pelo Comitê de Ética em Pesquisa do Centro Universitário de Maringá-UniCesumar em 20/11/2014 (Protocolo no 37086014.3.0000.5539, parecer no 879.619). Todos os responsáveis assinaram o Termo de Consentimento Livre e Esclarecido em nome de suas meninas. $\mathrm{O}$ estudo foi financiado pelo Centro Nacional de Desenvolvimento Científico e Tecnológico (CNPq) através do Programa Institucional de Bolsas de Iniciação Científica (PIBIC).

Foram incluídas no estudo meninas cadastradas em UBS distribuídas estrategicamente pelo município de Maringá, PR, de acordo com os estratos sociodemográficos - alvo da primeira campanha de vacinação contra HPV em 2014 (com idade entre 11-13 anos em 2014) - que não tomaram a $1^{\text {a }}$ e/ou a $2^{\mathrm{a}}$ dose da vacina dentro do prazo estipulado. ${ }^{15}$ Para determinar o total de meninas que preenchiam o 
critério estabelecido foi realizado um levantamento a partir do software Gestor Saúde. Esse software foi implementado na rede pública de saúde de Maringá no ano de 2008 pela Secretaria de Saúde do município e fornece, entre outros dados, informações sobre as vacinas administradas aos usuários. ${ }^{25}$

Foram identificadas um total de 274 meninas. Destas, 161 compuseram a amostra e 58 participaram efetivamente da pesquisa. O tamanho da amostra foi calculado pelo método da amostragem estratificada proporcional, considerando o número de meninas cadastradas em cada UBS. Para seleção daquelas que deveriam pertencer a amostra dentro de cada estrato, utilizou-se a amostragem aleatória simples.

Para coleta de dados, foi desenvolvido um questionário baseado em outros já validados, ${ }^{17,26-31}$ adaptado para a realidade local, abrangendo os seguintes aspectos: 1) perfil socioeconômico da população alvo; 2) nível de conhecimento acerca do HPV e da vacina; e 3) motivos para a recusa da vacina. Esse instrumento foi aplicado por estudantes de graduação, previamente capacitados, por meio de entrevistas face a face. A coleta ocorreu em um único momento na residência das meninas, com a permissão dos seus responsáveis.

A organização e sumarização dos questionários aplicados foi realizada em planilha do Microsoft Excel 2011. Os dados foram analisados através de estatística descritiva das variáveis, por meio de gráficos e tabelas de frequência, com auxílio do ambiente estatístico R.

\section{Resultados}

Os questionários foram aplicados a 58 (36\%) das 161 adolescentes elegíveis para o estudo. Os motivos que inviabilizaram sua aplicação para as demais adolescentes foram: 1) problemas em relação ao endereço (errado, incompleto, não disponível ou desatualizado) (45\%); 2) constatação no momento da visita de que a vacina já havia sido administrada (30\%); 3) adolescente não se encontrava em casa no momento da visita (22\%); e 4) recusa em participar da pesquisa (3\%).

Perfil socioeconômico da população-alvo

Observa-se pela Tabela 1 que a maioria das jovens questionadas (60\%) tem entre 12 e 13 anos, sendo que a idade média é de 12,6 anos. Em relação à idade dos responsáveis pelas adolescentes, apenas uma pequena parcela possui menos de 30 anos e mais que 50 (14\% e $5 \%$, respectivamente), ao passo que grande parte concentra-se na faixa etária de 30 a 40 anos. $86 \%$ das meninas estão cursando o ensino fundamental e $13 \%$, o ensino médio.

Já entre os responsáveis das meninas que não se vacinaram contra o HPV pode-se verificar que cerca de $46 \%$ deles possuem apenas o ensino fundamental, completo ou incompleto. Por outro lado, quase $21 \%$ cursou o ensino superior ou técnico. Também é interessante ressaltar que não houve registro de responsáveis analfabetos. A maioria das famílias das jovens questionadas (62\%) possui renda familiar entre 1 e 5 salários mínimos e apenas 15\% tem renda familiar superior a 5 salários mínimos.

Nota-se pela distribuição de frequências apresentas na Tabela 1, que mais da metade das meninas que responderam ao questionário (53\%) apontou ser da raça "branca", enquanto $41 \%$ e $5 \%$ são da raça "afrodescendente" e "amarela", respectivamente. Quase $50 \%$ das adolescentes que não tomaram vacina contra o vírus HPV e foram entrevistadas responderam que seguem a religião católica, 39\% evangélica e $1 \%$ segue outra religião. Ainda, $12 \%$ afirmaram não ter nenhuma religião. 
Tabela 1. Distribuição de frequências das respostas referentes à caracterização das adolescentes do município de Maringá, $\mathrm{PR}$, que não tomaram vacina contra HPV.

\begin{tabular}{|c|c|c|}
\hline Variáveis & Frequência & $(\%)$ \\
\hline 11 anos & 10 & $(17)$ \\
\hline 13 anos & 18 & (31) \\
\hline 14 anos & 13 & $(22)$ \\
\hline$<30$ anos & 8 & $(14)$ \\
\hline $30-40$ anos & 25 & $(43)$ \\
\hline $40-50$ anos & 22 & $(38)$ \\
\hline$\geq 50$ anos & 3 & $(5)$ \\
\hline EF cursando & 50 & $(86)$ \\
\hline EM cursando & 8 & $(14)$ \\
\hline \multicolumn{3}{|c|}{ Escolaridade do responsável $(n=58)$} \\
\hline Analfabeto & 0 & (0) \\
\hline EF incompleto & 22 & (38) \\
\hline EF completo & 5 & (9) \\
\hline EM incompleto & 5 & (9) \\
\hline EM completo & 13 & (22) \\
\hline Ensino superior & 5 & (9) \\
\hline Não soube responder & 6 & $(10)$ \\
\hline \multicolumn{3}{|l|}{ Raça $(n=58)$} \\
\hline Branca & 31 & (53) \\
\hline Afrodescendente & 24 & $(41)$ \\
\hline Amarela & 3 & (5) \\
\hline \multicolumn{3}{|l|}{ Religião ( $n=58$ ) } \\
\hline Católica & 27 & $(47)$ \\
\hline Evangélica & 23 & $(40)$ \\
\hline Nenhuma & 7 & (12) \\
\hline Outra & 1 & $(2)$ \\
\hline
\end{tabular}

EF: ensino fundamental; EM: ensino médio. Fonte: Dados da pesquisa. 
Nível de conhecimento acerca do vírus e da vacina

A Tabela 2 resume as informações sobre o conhecimento das meninas que responderam ao questionário sobre o vírus HPV, assim como a vacina contra ele. Observou-se que a grande maioria (86\%) já ouviu falar sobre o HPV. Também é possível verificar que pouco menos da metade delas (48\%) respondeu que existe relação entre a infecção pelo HPV e o câncer de colo de útero. Já quando questionadas sobre a existência de relação entre a infecção pelo HPV e o desenvolvimento de verrugas genitais, quase $60 \%$ das adolescentes responderam afirmativamente.

Nota-se também que $70 \%$ das jovens acreditam que há relação entre o tabagismo e o câncer de colo de útero. Ainda, mais de $60 \%$ disse acreditar na relação entre a infecção pelo HPV e a idade de iniciação sexual, enquanto quase $75 \%$ apontou que, em sua opinião, existe relação entre a infecção e a quantidade de parceiros sexuais. $74 \%$ das meninas questionadas apontaram a relação sexual como meio de transmissão do HPV e 10\% o compartilhamento de copos e talheres, ao passo que os fatores aerossol e compartilhamento de toalhas de banho foram citados cada um por $5 \%$ das adolescentes em estudo; e picada de mosquito por apenas $3 \%$.

Verifica-se ainda que $62 \%$ das adolescentes que participaram da pesquisa responderam de maneira afirmativa a existência de cura para a infecção pelo HPV, sendo que a maioria das entrevistadas (88\%) apontou que já ouviu falar sobre a vacina contra o HPV.

Motivos para a recusa do benefício

As 58 meninas entrevistadas também foram questionadas sobre as razões pelas quais deixaram de tomar a vacina que protege contra o vírus HPV. A distribuição de frequências das respostas encontra-se disposta na Tabela 3. O desconhecimento sobre o vírus foi citado por apenas $7 \%$ das adolescentes como motivo para não se vacinarem, enquanto o desconhecimento da vacina impediu que $8 \%$ delas procurassem o benefício. Quase 14\% citaram a falta de conhecimento da campanha nacional de vacinação.

O motivo apontado com maior frequência foi o medo quanto aos efeitos colaterais, seguido pela impossibilidade de ir até UBS/Secretaria de Saúde para vacinar-se (37\% e 20\%, respectivamente). 13\% das jovens não se vacinaram devido ao medo de injeção. Pela Tabela 3, fica evidente que a recusa por parte do responsável foi uma justificativa frequente para as adolescentes não se vacinarem, destacando a preocupação quanto aos efeitos colaterais, que foi apontado por $17 \%$ delas.

Ainda considerando a recusa do responsável, a crença religiosa e de que a vacina possa promover promiscuidade, foi apontado, cada um, apenas por uma adolescente entrevistada. A ausência de um motivo aparente para a recusa da vacina foi descrita como "outro" na Tabela 3.

\section{Discussão}

A maior parte das adolescentes deste estudo, felizmente, já ouviu falar do Papilomavírus Humano, no entanto, ideias errôneas quanto a sua transmissão, sua relação com o câncer de colo de útero e quanto à cura da infecção são comuns. Quinze das 58 (26\%) adolescentes não acreditam que a via sexual seja a 
Tabela 2. Distribuição de frequências das respostas referentes aos conhecimentos sobre o HPV/vacina das adolescentes do município de Maringá, PR, que não tomaram vacina contra HPV.

\begin{tabular}{|c|c|c|}
\hline Perguntas & Frequência & $(\%)$ \\
\hline \multicolumn{3}{|l|}{ Já ouviu falar em HPV? (n=58) } \\
\hline $\operatorname{Sim}$ & 50 & (86) \\
\hline \multicolumn{3}{|l|}{ Como é transmitido o HPV? $(n=58)$} \\
\hline Aerossol & 3 & (5) \\
\hline Relação Sexual & 43 & $(74)$ \\
\hline Compartilhamento de copos e talheres & 6 & $(10)$ \\
\hline Compartilhamento de toalhas de banho & 3 & (5) \\
\hline Não soube responder & 1 & (2) \\
\hline
\end{tabular}

Existe relação entre a infecção pelo HPV e o CA de colo de útero? ( $n=58)$

$\begin{array}{lrr}\text { Sim } & (48) \\ \text { Não } & 28 & (50) \\ \text { Não soube responder } & 29 & 1 \\ \end{array}$

Existe relação entre a infecção pelo HPV e o desenvolvimento de verrugas genitais? $(n=58)$

$\begin{array}{lrr}\text { Sim } & 34 & (58) \\ \text { Não } & 21 & (37) \\ \text { Não soube responder } & 3 & (5)\end{array}$

Acredita que há relação entre o tabagismo e o desenvolvimento de CA de colo de útero? (n=58)

Sim 41

$41 \quad(71)$

Não

Não soube responder

1

\begin{tabular}{|c|c|c|}
\hline Sim & 37 & $(64)$ \\
\hline Não & 18 & $(31)$ \\
\hline \multicolumn{3}{|c|}{ Acredita que há relação entre a infecção pelo HPV e a quantidade de parceiros sexuais? $(n=58)$} \\
\hline Sim & 43 & $(74)$ \\
\hline Não soube responder & 3 & (5) \\
\hline \multicolumn{3}{|c|}{ Há cura para a infecção pelo HPV? (n=58) } \\
\hline Sim & 36 & $(62)$ \\
\hline Não & 19 & (33) \\
\hline Não soube responder & 3 & (5) \\
\hline
\end{tabular}

HPV: Papilomavírus Humano; CA: câncer. Fonte: dados da pesquisa. 
Tabela 3. Distribuição de frequências dos motivos pelos quais as adolescentes no município de Maringá, PR, não tomaram a vacina contra o HPV.

\begin{tabular}{|c|c|c|}
\hline Motivos & Frequência* & $(\%)$ \\
\hline Desconhecimento do vírus & 4 & (7) \\
\hline Desconhecimento da vacina contra o HPV & 5 & (9) \\
\hline Desconhecimento da campanha nacional de vacinação contra o HPV & 8 & $(14)$ \\
\hline Medo de injeção & 8 & $(14)$ \\
\hline Medo de efeitos colaterais da vacina contra o HPV & 22 & $(38)$ \\
\hline Não teve como ir à UBS/Secretaria de Saúde para vacinar-se & 12 & $(21)$ \\
\hline \multicolumn{3}{|l|}{ Recusa por parte do responsável: } \\
\hline Necessidade de maiores informações sobre o vírus/vacina & 4 & $(7)$ \\
\hline Preocupações quanto à segurança da vacina & 4 & $(7)$ \\
\hline Preocupações quanto a efeitos colaterais & 10 & $(17)$ \\
\hline Acredita que a vacina possa promover promiscuidade & 1 & $(2)$ \\
\hline Acredita que a vacina possa promover início precoce da atividade sexual & 5 & (9) \\
\hline Acredita que sua filha não precisa da vacina por ser muito nova/não ser sexualmente ativa & 2 & (3) \\
\hline Crença religiosa & 1 & $(2)$ \\
\hline Necessidade de discutir sobre DST com a filha & 0 & (0) \\
\hline Outro & 1 & $(2)$ \\
\hline Outro & 4 & $(7)$ \\
\hline
\end{tabular}

${ }^{*}$ Neste caso, a soma das frequências observadas em todas as alternativas é superior ao total, uma vez que era possível optar por mais de uma alternativa. HPV: Papilomavírus Humano; UBS: Unidade Básica de Saúde; DST: doença sexualmente transmissível. Fonte: dados da pesquisa.

forma de transmissão do vírus, 30 (52\%) desconhecem a íntima relação entre a infecção pelo HPV e o câncer de colo de útero enquanto 24 (41\%) não conhecem a relação com o desenvolvimento de verrugas genitais.

Os fatores de risco conhecidos para a infecção pelo HPV são início precoce da atividade sexual; número de parceiros sexuais e manter relação com parceiro que tenha outros parceiros, tanto homens quanto mulheres. ${ }^{32,33}$ Vinte e uma entrevistadas (36\%) não acreditam que o início precoce da atividade sexual tenha relação com a infecção pelo vírus, enquanto $12(21 \%)$ delas não sabem da influência de múltiplos parceiros sexuais. Por fim, 40 adolescentes (69\%) creem que há cura para a infecção, o que não é verdadeiro.

Estes resultados estão em concordância com pesquisas internacionais, que mostraram que adultos e adolescentes pouco sabem sobre o HPV, não só em países em desenvolvimento como também nos desenvolvidos. ${ }^{18,19,33,34}$ No Reino Unido, cerca de $30 \%$ da população feminina já ouviu falar no vírus; no Canadá, cerca de 13\% das adolescentes; e nos Estados Unidos, apenas 37\% das mulheres e, entre aquelas que o conhecem de fato, dúvidas e ideias errôneas ainda são comuns. ${ }^{35}$

Grande variação nos índices de cobertura vacinal nesses países pode ser reflexo do pouco conhecimento acerca do HPV e da sua vacina, como indicado pelas pesquisas citadas; por outro lado, países com melhor nível socioeducacional têm a cultura do questionamento, gerando um comportamento mais maduro frente ao assunto.

No Canadá, os dados mais recentes trazem que a adesão à vacina contra o HPV varia de 47 a $94 \%$ entre as províncias; ${ }^{36}$ nos EUA, 32\% das adolescentes entre 13 e 17 anos completaram o esquema de três doses. ${ }^{37}$ Fugindo deste padrão, o Reino Unido vem obtendo mais sucesso nas campanhas de vacinação 
contra o HPV, imunizando $76 \%$ da população-alvo. ${ }^{37}$ Algo a ser considerado na pesquisa atual é o formato do questionário empregado, que por ser composto de perguntas fechadas pode ter influenciado a resposta das entrevistadas sem necessariamente refletir o conhecimento acerca do HPV. Este é um possível viés do estudo, devendo ser considerado em trabalhos futuros.

Inúmeras pesquisas foram realizadas na África, na Ásia, na América Latina, na Europa, na Austrália e nos Estados Unidos a fim de se avaliar os fatores de resistência de pais frente a vacina anti-HPV, antes e depois de ser liberada nesses países, com o intuito de alcançar altas taxas de imunização a partir de campanhas publicitárias eficientes que abrangessem as dúvidas dos cidadãos, ${ }^{26-31,37-42}$ uma vez que a oposição dos pais é o maior obstáculo para a vacinação de crianças contra o HPV. ${ }^{20,43}$ Esta pesquisa traz a recusa por parte dos pais como fator importante na adesão à campanha de vacinação, porém não preponderante, destacando-se, portanto, o medo de efeitos colaterais da vacina contra o HPV.

Apesar de os motivos que levaram à recusa da vacina serem praticamente os mesmos em todo o mundo, identificou-se nessas pesquisas que preocupações mais pragmáticas, como possíveis efeitos colaterais; segurança da vacina; necessidade de maiores informações acerca do HPV, do câncer cervical e da vacina; são comuns entre moradores de países desenvolvidos, de maior nível socioeconômico e educacional, como a Austrália, os EUA, o Canadá e países europeus, como Holanda, Espanha, Suécia e França. ${ }^{17,21-24,26,27,30,31,42}$

Preocupações morais e religiosas acometem moradores de países subdesenvolvidos, como africanos, latinos e asiáticos; e são exemplos: idade imprópria para se pensar em sexo; aguardar a filha ter idade suficiente para tomar a decisão por si só, já que não querem se sentir responsáveis por qualquer efeito negativo da vacina sobre ela; vergonha de tomar vacina contra uma DST e como religiões pregam a abstinência sexual até o casamento, a vacina torna-se desnecessária para os religiosos. ${ }^{20,21,23,29,30,36,39}$ Questões como negação da necessidade de vacinação, temor em discutir sobre sexo e DSTs com suas filhas e a preocupação quanto a vacina levar à promiscuidade foram abordadas por pais de todas as nacionalidades, credos e raças. .2,26,27,29,42 $^{2}$

A pesquisa atual traz dados diferentes. Questões pragmáticas foram as mais comentadas neste estudo, apesar de estarmos em um país subdesenvolvido e a população entrevistada pertencer à classe social baixa, com renda familiar entre 1 e 5 salários mínimos. As questões mais citadas foram o medo de efeitos colaterais, seguido de preocupações quanto a segurança da vacina e necessidade de maiores informações sobre o vírus/vacina. Preocupações morais e religiosas também foram discutidas, porém em menor proporção.

Tanto por parte dos pais quanto das meninas, o medo de efeitos colaterais foi preponderante na tomada de decisão. Durante a aplicação dos questionários, foi inquerido o motivo desta preocupação, e todas as adolescentes se referiram ao caso de uma jovem da região que apresentou parestesia de membros inferiores seis meses após tomar a vacina, fato relacionado, por ela, à vacina. Uma entrevistada relatou se preocupar com infertilidade.

Algumas pesquisas já foram realizadas acerca deste tema e a mais recente, que estudou 189.629 mulheres entre 9 e 26 anos que tomaram pelo menos uma dose da vacina quadrivalente no período entre 2006 e 2008, concluiu que efeitos colaterais são raros e benignos, sendo os mais comuns a síncope logo 
após a tomada da vacina e reações no local de administração em até 2 semanas. ${ }^{44} \mathrm{~A}$ associação entre a vacina e a síncope já era esperada, uma vez que esse efeito é comum a qualquer vacina, principalmente na faixa etária estudada; as reações de pele no sítio da aplicação, como dor e edema, possuem efeito passageiro.

Em 2013, a International Federation of Gynecology and Obstetrics (FIGO) publicou um pronunciamento acerca da segurança desta vacina, indicando que todos os seus ensaios clínicos provaram grande segurança na administração tanto da vacina bivalente quanto da quadrivalente, e que o efeito colateral mais referido foi a dor limitada ao local da administração e de resolução espontânea. ${ }^{45}$

Eventos raros e temidos são o tromboembolismo venoso, doenças autoimunes e doenças desmielinizantes. Frente a isso, entidades como o US Centers for Disease Control and Prevention (CDC); e a Organização Mundial da Saúde (OMS), baseada nas recomendações do The Global Advisory Comittee on Vaccine Safety (GACVS) desenvolveram relatórios em 2014 e 2015, respectivamente, em que não comprovam qualquer relação causal entre essas condições e a vacina Anti-HPV e mantêm a indicação da vacina. ${ }^{46-48}$ Doenças imunomediadas vêm sendo associadas principalmente aos adjuvantes presentes nas vacinas em geral, como o hidróxido de alumínio quando nos referimos à vacina contra o HPV. Geralmente, aqueles que desenvolvem quadros autoimunes possuem já fatores predisponentes. ${ }^{49}$

Outros motivos bastante comentados que impossibilitaram a tomada da vacina foram o desconhecimento do vírus, da vacina, ou mesmo da campanha nacional de vacinação contra o HPV, fatores que reforçam a importância de se realizar programas de educação e conscientização da população sobre o vírus e a vacina junto à campanha de imunização, a fim de se aumentar a adesão à vacina. Embora neste trabalho a população investigada não tenha questionado a efetividade da vacina, este é um fator importante a ser considerado em estudos próximos.

A recusa por parte dos pais influencia fortemente a decisão das adolescentes, que geralmente concordam com eles. ${ }^{20,35}$ Dessa forma, a influência sociocultural ainda representa um grande empecilho para o sucesso da implementação do programa de vacinação, não só no município de Maringá, PR, mas em todo o mundo, que já tem como exemplo a Grécia, os Estados Unidos da América e o Reino Unido, que já completaram o esquema de três doses da vacina, cobrindo não mais que $9 \%, 32 \%$ e $76 \%$, respectivamente, da meta estipulada. ${ }^{37}$

Independentemente do sucesso da implementação da vacina contra o HPV, exames de rotina devem ser realizados periodicamente com o intuito de se detectar lesões precursoras de câncer cervical logo no início do seu desenvolvimento, tratando-as e prevenindo a progressão para o câncer de colo de útero. $O$ Papanicolaou é o principal método de rastreamento, tanto da infecção pelo HPV quanto do câncer. ${ }^{50}$

O presente estudo, apesar de sua amostra limitada, coloca em pauta a necessidade de divulgar à população geral, por meio de campanhas publicitárias e dentro das salas de aula, informações sobre a infecção pelo HPV, possíveis benefícios e efeitos colaterais da vacina, como pontos fundamentais para o sucesso de uma próxima campanha de vacinação contra o HPV. 


\section{Referências}

1. Centers for Disease Control and Prevention (CDC). National Center for HIV/AIDS, Viral Hepatitis, STD, and TB Prevention. Division of STD Prevention Genital HPV. Genital HPV Infection - Fact Sheet [acesso 2017 Ago 12]. Disponível em: http://www.cdc.gov/std/HPV/ STDFact-HPV.htm

2. Stanley M. Pathology and epidemiology of HPV infection in females. Gynecol Oncol. 2010;117(2 Suppl):S5-10. DOI: http://dx.doi. org/10.1016/j.ygyno.2010.01.024

3. Girianelli VR, Thuler LCS, Azevedo e Silva G. Prevalência de HPV em mulheres assistidas pela Estratégia Saúde da Família na Baixa Fluminense do Estado do Rio de Janeiro. Rev Bras Ginecol Obstet. 2010;32(1):39-46. DOI: http://dx.doi.org/10.1590/S010072032010000100007

4. Clifford GM, Gallus S, Herrero R, Muñoz N, Snijders P, Vacarella S, et al.; IARC HPV Prevalence Surveys Study Group. Worldwide distribution of human papillomavirus types in cytologically normal women in the International Agency for Research on Cancer HPV prevalence surveys: a pooled analysis. Lancet. 2005;366(9490):991-8. DOI: http://dx.doi.org/10.1016/S0140-6736(05)67069-9

5. World Health Organization. International Agency for Research on Cancer. Globocan 2012: Estimated Cancer Incidence, Mortality and Prevalence Worldwide In 2012 [acesso 2017 Ago 12]. Disponível em: http://Globocan.larc.Fr/Pages/Fact_Sheets_Population.Aspx

6. Brasil. Instituto Nacional de Câncer. Atlas On-Line de Mortalidade [acesso 2017 Ago 12]. Disponível em: https://mortalidade.inca.gov. br/MortalidadeWeb/pages/Modelo04/consultar.xhtml\#paneIResultado

7. Parkin D, Bray F. Chapter 2: The burden of HPV-related cancers. Vaccine. 2006;24 Suppl 3:S3/11-25. DOI: http://dx.doi.org/10.1016/j. vaccine.2006.05.111

8. Park IU, Introcaso C, Dunne EF. Human Papillomavirus and Genital Warts: A Review of the Evidence for the 2015 Centers for Disease Control and Prevention Sexually Transmitted Diseases Treatment Guidelines. Clin Infect Dis. 2015;61 Suppl 8:S849-55. DOI: http://dx.doi. org/10.1093/cid/civ813

9. Bayas JM, Costas L, Muñoz A. Cervical cancer vaccination indications, efficacy, and side effects. Ginecol Oncol. 2008;110(3 Suppl 2):S11-4. DOI: http://dx.doi.org/10.1016/j.ygyno.2008.05.011

10. Sanches EB. Prevenção do HPV: a utilização da vacina nos serviços de saúde. Saude e pesqui. 2011;3(2):255-61.

11. Kasting ML, Wilson S, Dixon BE, Downs SM, Kulkarni A, Zimet GD. A qualitative study of healthcare provider awareness and informational needs regarding the nine-valent HPV vaccine. Vaccine. 2016;11(34):1331-4. DOI: http://dx.doi.org/10.1016/j.vaccine.2016.01.050

12. Human Papillomavirus Vaccines: WHO Position Paper, October 2014. Wkly Epidemiol Rec. 2014;89(43):465-92.

13. Denny L. Cervical cancer prevention: new opportunities for primary and secondary prevention in the 21 st century. Int J Gynecol Obstet. 2012;119 Suppl 1:S80-4. DOI: http://dx.doi.org/10.1016/j.ijgo.2012.03.023

14. Brasil. Ministério da Saúde [Internet]. Mais de 158 mil meninas já foram vacinadas contra HPV no Paraná; 2014 [acesso 2017 Ago 12]. Disponível em: http://portalsaude.saude.gov.br/index.php?option=com_content\&view=article\&id=11984\&catid=11\&ltemid=103

15. Portal Brasil. Vacinação contra o HPV começa nesta segunda-feira (10): Meninas de 11 a 13 anos serão vacinadas. Elas devem receber três doses da vacina. A segunda após seis meses e a terceira após 5 anos. 7 de março de 2014 [acesso 2017 Ago 12]. Disponível em: http://www.brasil.gov.br/saude/2014/03/vacinacao-contra-hpv-comeca-nesta-segunda-feira-10

16. G1 Maringá (Maringá). Maringá já vacinou 52,4\% do público alvo contra o HPV, informa secretaria: Mais de 3,9 mil meninas de 11 a 13 anos já foram vacinadas no município. Meta é chegar a 7,5 mil imunizadas até o fim da campanha. 22 de abril 2014 [acesso 2014 Abr 8]. Disponível em: http://g1.globo.com/pr/norte-noroeste/noticia/2014/04/maringa-ja-vacinou-524-do-publico-alvo-contra-o-hpv-informasecretaria.html

17. Toffolon-Weiss M, Hagan K, Leston J, Peterson L, Provost E, Henessy T. Alaska Native parental attitudes on cervical cancer, HPV and the HPV vaccine. Int J Circumpolar Health. 2008;67(4):363-73. DOI: http://dx.doi.org/10.3402/ijch.v67i4.18347

18. LaMontagne DS, Barge S, Le NT, Mugisha E, Penny ME, Ghandi S, et al. Human papillomavirus vaccine delivery strategies that achieved high coverage in low- and middle-income countries. Bull World Health Organ. 2011;89(11):821-30B. DOI: http://dx.doi.org/10.2471/ BLT.11.089862 
19. Phan DP, Pham QT, Strobel M, Tran DS, Tran TL, Buisson Y. Acceptabilité de la vaccination contre les papillomavirus humains (HPV) par les pédiatres, les mères et les jeunes femmes à Hô Chi Minh Ville, Vietnam. Rev Epidemiol Sante Publique. 2012;60(6):437-46. DOI: http://dx.doi.org/10.1016/j.respe.2012.03.010

20. Craciun C, Baban A. "Who will take the blame?": Understanding the reasons why Romanian mothers decline HPV vaccination for their daughters. Vaccine. 2012;30(48):6789-93. DOI: http://dx.doi.org/10.1016/j.vaccine.2012.09.016

21. Grandahl M, Oscarsson M, Stenhammar C, Nevéus T, Westerling R, Tydén T. Not the right time: why parents refuse to let their daughters have the human papillomavirus vaccination. Acta Pædiatr. 2014;103(4):436-41. DOI: http://dx.doi.org/10.1111/apa.12545

22. Krawczyk A, Perez S, King L, Vivion M, Dubé E, Rosberger Z. Parents' decision-making about the human papillomavirus vaccine for their daughters: II. Qualitative results. Hum Vaccin Immunother. 2015;11(2):330-6. DOI: http://dx.doi.org/10.4161/21645515.2014.980708

23. Navarro-illana P, Caballero P, Tuells J, Puig-Barberá J, Diez-Domingo J. Aceptabilidad de la vacuna contra el virus del papiloma humano en madres de la provincia de Valencia (España). An Pediatr (Barc.). 2015;83(5):318-27.

24. Sabiani L, Bremond A, Mortier I, Lecuyer M, Boubli L, Carcopino X. Évaluation de la couverture vaccinale du vaccin anti-hpv: résultats d'une enquête auprès des lycéennes et étudiantes de la région PACA. J Gynecol Obstet Biol Reprod (Paris). 2012;41(2):136-44. DOI: http://dx.doi.org/10.1016/j.jgyn.2011.10.001

25. Prefeitura de Maringá. Diretor do Datasus elogia Sistema de Gestão de Saúde de Maringá. 21 de março de 2011 [acesso 2015 Dez 1]. Disponível em: http://www2.maringa.pr.gov.br/site/imprensa/impressao.php?id=12905

26. Marshall H, Ryan P, Roberton D, Baghurst P. A cross-sectional survey to assess community attitudes to introduction of Human Papillomavirus vaccine. Aust N Z J Public Health. 2007;31(3):235-42. DOI: http://dx.doi.org/10.1111/j.1467-842X.2007.00054.x

27. Constantine NA, Jerman P. Acceptance of human papillomavirus vaccination among Californian parents of daughters: a representative statewide analysis. J Adolesc Health. 2007;40(2):108-15. DOI: http://dx.doi.org/10.1016/j.jadohealth.2006.10.007

28. Yaganeh N, Curtis D, Kuo A. Factors influencing HPV vaccination status in a Latino population; and parental attitudes towards vaccine mandates. Vaccine. 2010;28(25):4186-91. DOI: http://dx.doi.org/10.1016/j.vaccine.2010.04.010

29. Chan SS, Cheung TH, Lo WK, Chung TK. Women's attitudes on human papillomavirus vaccination to their daughters. J Adolesc Health. 2007;41(2):204-7. DOI: http://dx.doi.org/10.1016/j.jadohealth.2007.04.011

30. Marlow LA, Wardle J, Forster AS, Waller J. Ethnic differences in human papillomavirus awareness and vaccine acceptability. J Epidemiol Community Health. 2009;63(12):1010-5. DOI: http://dx.doi.org/10.1136/jech.2008.085886

31. Lenselink CH, Gerrits MM, Melchers WJ, Massuger LF, van Hamont D, Bekkers RL. Parental acceptance of Human Papillomavirus vaccines. Eur J Obstet Gynecol Reprod Biol. 2008;137(1):103-7. DOI: http://dx.doi.org/10.1016/j.ejogrb.2007.02.012

32. Wheeler CM. The natural history of cervical human papillomavirus infections and cervical cancer: gaps in knowledge and future horizons. Obstet Gynecol Clin North Am. 2013;40(2):165-76. DOI: http://dx.doi.org/10.1016/j.ogc.2013.02.004

33. Sousa LB, Pinheiro AK, Barroso MG. Ser mulher portadora do HPV: uma abordagem cultural. Rev Esc Enferm USP. 2008;42(4):737-43. DOI: http://dx.doi.org/10.1590/S0080-62342008000400017

34. Costa LA, Goldenberg P. Papilomavírus humano (HPV) entre jovens: um sinal de alerta. Saúde Soc. 2013;22(1)249-61. DOI: http://dx.doi. org/10.1590/S0104-12902013000100022

35.Zimet GD. Improving adolescent health: focus on HPV vaccine acceptance. J Adolesc Health. 2005;37(6 Suppl):S17-23. DOI: http:// dx.doi.org/10.1016/j.jadohealth.2005.09.010

36. Canadian Partnership Against Cancer. The 2015 Cancer System Performance Report. Toronto: Canadian Partnership Against Cancer; 2015. $161 \mathrm{p}$.

37. Remes P, Selestine V, Changalucha J, Ross DA, Wight D, de Sanjosé S, et al. A qualitative study of HPV vaccine acceptability among health workers, teachers, parents, female pupils, and religious leaders in northwest Tanzania. Vaccine. 2012;36(30):5363-7. DOI: http:// dx.doi.org/10.1016/j.vaccine.2012.06.025

38. Sperber NR, Brewer NT, Smith JS. Influence of parent characteristics and disease outcome framing on HPV vaccine acceptability among rural, Southern women. Cancer Causes Control. 2008;19(1):115-8. DOI: http://dx.doi.org/10.1007/s10552-007-9074-9

39. Read DS, Joseph MA, Polishchuck V, Suss AL. Attitudes and Perceptions of the HPV Vaccine in Caribbean and African-American Adolescent Girls and their Parents. J Pediatr Adolesc Gynecol. 2010;23(4):242-5. DOI: http://dx.doi.org/10.1016/j.jpag.2010.02.002

40. Bartolini RM, Winkler JL, Penny ME, LaMontagne DS. Parental acceptance of HPV vaccine in Peru: a decision framework. PLoS One. 2012;7(10):e48017. DOI: http://dx.doi.org/10.1371/journal.pone.0048017 
41. Dinh TA, Rosenthal SL, Doan ED, Trang T, Pham VH, Tran BD, et al. Attitudes of mothers in Da Nang, Vietnam toward a human papillomavirus vaccine. J Adolesc Health. 2007;40(6):559-63. DOI: http://dx.doi.org/10.1016/j.jadohealth.2007.02.003

42. Brabin L, Roberts SA, Farzaneh F, Kitchener HC. Future acceptance of adolescent human papillomavirus vaccination: a survey of parental attitudes. Vaccine. 2006;24(16):3087-94. DOI: http://dx.doi.org/10.1016/j.vaccine.2006.01.048

43. Zimet GD, Liddon N, Rosenthal SL, Lazcano-Ponce E, Allen B. Psychosocial aspects of vaccine acceptability. Vaccine. 2006;24 Suppl 3:S3/201-209. DOI: http://dx.doi.org/10.1016/j.vaccine.2006.06.017

44. Klein NP, Hansen J, Chao C, Velicer C, Emery M, Slezak J, et al. Safety of quadrivalent human papillomavirus vaccine administered routinely to females. Arch Pediatr Adolesc Med. 2012;166(12):1140-8. DOI: http://dx.doi.org/10.1001/archpediatrics.2012.1451

45. FIGO - International Federation of Gynecology and Obstetrics. Safety of HPV vaccination: a FIGO Statement; 2013 [acesso 2017 Ago 12]. Disponível em: http://www.figo.org/sites/default/files/uploads/project-publications/Statement on Safety of HPV vaccination - FINAL - AUGUST 2013_0.pdf

46. Vichnin M, Bonanni P, Klein NP, Garland SM, Block SL, Kjaer SK, et al. An Overview of Quadrivalent Human Papillomavirus Vaccine Safety. Pediatr Infect Dis J. 2015;34(9):983-91. DOI: http://dx.doi.org/10.1097/INF.0000000000000793

47.21 Global Advisory Committee on Vaccine Safety, 2-3 December 2015. 31 Monthly report on dracunculiasis cases, January-November 2015. Wkly Epidemiol Rec. 2016;91(3):21-32. DOI: http://dx.doi.org/10.1177/003335491513000606

48. White MD. Pros, cons, and ethics of HPV vaccine in teens-Why such controversy? Transl Androl Urol. 2014;3(4):429-34.

49. Jara LJ, García-Collinot G, Medina G, Cruz-Dominguez MDP, Vera-Lastra O, Carranza-Muleiro RA, et al. Severe manifestations of autoimune syndrome induced by adjuvants (Shoenfeld's syndrome). Immunol Res. 2017;65(1):8-16. DOI: http://dx.doi.org/10.1007/ s12026-016-8811-0

50. Berek JS. Berek e Novak - Tratado de Ginecologia. Rio de Janeiro: Guanabara Koogan; 2014.

a Centro Universitário Cesumar (UniCesumar). Maringá, PR, Brasil. zanini91@ hotmail.com; bianca.stawinski@gmail.com; rafa_hendges@ hotmail.com; krou_arnaut@icloud.com; marcelo.bernuci@unicesumar.edu.br (Autor correspondente)

b Universidade Federal de São Carlos (UFSCAR). São Carlos, SP, Brasil. fercallegari@ufscar.br 